\title{
Autopercepção de saúde dos motoristas de transporte coletivo
}

\author{
Health self-perception of public transport drivers
}

\author{
1 Juliana Ladeira Garbaccio julianapuciec@gmail.com \\ 2 Iury Augusto Otoni Pereira
}

\footnotetext{
1 Odontóloga e enfermeira. Docente da PucMinas. Mestre em microbiologia e doutora em Enfermagem. Especialista em Enfermagem Gerontologica, Enfermagem em Dermatologia. Pontificia Universidade Católica de Minas Gerais.

2 Acadêmico de Enfermagem. Pontifícia Universidade Católica de Minas Gerais.
}

\section{Resumo}

O objetivo desta pesquisa é avaliar a autopercepção de saúde dos motoristas de ônibus urbanos. Trata-se de um estudo transversal com 324 motoristas de transporte coletivo de Belo Horizonte/Minas Gerais, por meio de questionário estruturado sobre perfil socioeconômico/ profissional, condições de saúde, hábitos alimentares, dados vitais e antropométricos dos motoristas. A variável independente autopercepção de saúde foi dicotomizada em "boa/ruim", com análises estatísticas pelos testes Qui-quadrado, Exato de Fisher e Regressão de Poison. Observou-se que a "boa" autopercepção de saúde foi referida por $72,8 \%$, sendo associada $(p<0,05)$ à ausência de doença crônica (78,1\%), não utilização de medicação contínua $(77,4 \%)$ e ausência de dores musculoesquelética (84,3\%). Na regressão, houve associação com não possuir hipertensão arterial sistêmica, dor de cabeça, dores em membros inferiores, estar satisfeito com o dono da empresa e não ser fumante. Conclui-se que os motoristas participantes da pesquisa apresentam uma boa autopercepção de saúde apesar de fatores laborais e ambientais desfavoráveis reconhecidos por eles. Ademais, foi possível concluir que os pesquisados possuem limitações na percepção de saúde e no conceito de saúde como ausência de doença.

\section{Palavras-chave:}

Condições de trabalho. Saúde do trabalhador. Condução de veículo.

\begin{abstract}
The objective of this research is to evaluate the selfperceived health of urban bus drivers. This is a crosssectional study with 324 public transport drivers from Belo Horizonte/Minas Gerais, using a structured questionnaire on socioeconomic/professional profile, health conditions, eating habits, vital and anthropometric data of drivers. The independent variable self-perceived health was dichotomized as "good / bad", with statistical analysis using the Chi-square, Fisher's Exact and Poison Regression tests. It was observed that "good" selfperceived health was reported by $72.8 \%$, being associated $(p<0.05)$ with the absence of chronic disease $(78.1 \%)$, non-use of continuous medication (77.4\%) and absence of musculoskeletal pain (84.3\%). In the regression, there was an association with not having systemic arterial hypertension, headache, pain in the lower limbs, being satisfied with the owner of the company and not being a smoker. It is concluded that the drivers participating in the research have a good self-perception of health despite the unfavorable work and environmental factors recognized by them. In addition, it was possible to conclude that the respondents have limitations in the perception of health and in the concept of health as the absence of disease.
\end{abstract}

\section{Keywords:}

Working conditions. Occupational health. Automobile driving.

\section{Como você deve citar?}

GARBACCIO, Juliana Ladeira. Autopercepção de saúde dos motoristas de transporte coletivo. Cadernos UniFOA, Volta Redonda, n. 45, p. 133-145, abril 2021. 


\section{INTRODUÇÃO}

O transporte coletivo de ônibus é um dos serviços mais utilizados pela população. Em cidades com mais de 60 mil habitantes, os ônibus chegam a atender $84 \%$ dos deslocamentos da população. Contudo, os motoristas, importantes na força de trabalho desse setor de transporte, muitas vezes passam despercebidos no que refere ao bem-estar e saúde (PINTO; NEVES, 2009; VASCONCELLOS, CARVALHO, PEREIRA, 2011).

Esses profissionais vivenciam no cotidiano inúmeros desafios que afetam diretamente a saúde mental, física e emocional, como o difícil trânsito das cidades, as condições das vias, estações climáticas, atrasos de viagens, a relação com os usuários que exigem paciência, empatia e atenção, para que consiga concluir o percurso definido (PINTO; NEVES, 2009).

Aliado a essas variáveis, o condutor passa por ocasiões que podem prejudicar a própria saúde, haja vista os ruídos do automóvel, as altas temperaturas, as vibrações decorrentes de corpo, risco de assaltos e agressões, estresse referentes a atrasos entre viagens ou cobranças por parte da chefia (ALVES; PAULA, 2009).

Episódios de violência são apresentados pela literatura com $45 \%$ de motoristas e cobradores de ônibus relatando-os em uma entrevista, sendo $87 \%$ dos episódios cometidos pelos próprios passageiros (ASSUNÇÃO; MEDEIROS, 2015). Motoristas ainda encontram dificuldade para realizarem refeições, visto que necessitam cumprir os horários sem atrasos, para que possam ter tempo para pausas e alimentação, com intervalo curto. Com isso, realizam menos refeições e acabam por optar por alimentos rápidos ou não saudáveis (PINTO; NEVES, 2009; MORAES; FAYH, 2011; SOUZA; OROZCO-SOLIS et al., 2017; ASSUNÇÃO; PIMENTA, 2019; ).

A partir do comprometimento da saúde, o profissional pode ser afastado de forma temporária ou indeterminada do trabalho, de acordo com danos sofridos. É garantido legalmenteo cuidado com a saúde dos trabalhadores, por meio da Lei Orgânica da Saúde (Lei n8080/1990), que define a saúde do trabalhador como um dos campos de atuação do Sistema Único de Saúde (SUS). Essa área deve receber ações da vigilância epidemiológica e sanitária, atividades para promoção, proteção, recuperação e reabilitação da saúde dos trabalhadores. Além disso, é garantindo a participação do SUS com pesquisas para averiguar os riscos envolvidos no processo de trabalho, advertir entidades sindicais e empresas quanto aos riscos de acidente e doenças do trabalho (BRASIL, 1990).

Diante disso, uma ótima forma de entender o processo de saúde e doença desses profissionais é por meio da autopercepção que possuem sobre sua própria saúde. Esse conceito se utiliza de critérios objetivos e subjetivos, sendo um importante preditor para indicadores de mortalidade e morbidade individual, afetando a qualidade de vida. Possui influências sociais, culturais, econômicas e biológicas em consonância com os aspectos multidimensionais da determinação de saúde que envolve os fatores psicossociais, espirituais e uma boa qualidade de trabalho (AZEVEDO; FRICHE; LEMOS, 2012; PAVÃO; WERNECK; CAMPOS, 2013). Assim pergunta-se: qual a autopercepção de saúde entre os motoristas de ônibus urbano dentrodo cotidiano de trabalho?

Este estudo justifica-se pelo fato de as condições de ambiente de trabalho do motorista de transporte coletivo serem desfavoráveis, tanto interna quanto externamente. Pelo modo e estruturas de trabalho dessa categoria, os trabalhadores estão vulneráveis a doenças e agravos relacionados a doenças crônicas, ergonômicas e psicológicas sendo necessária a melhora do ambiente de trabalho (PINTO; NEVES, 2009; ASSUNÇÃO; SILVA, 2013; DE VITTA et al., 2013). 
Nesse sentido, esta pesquisa teve como objetivo avaliar a autopercepção de saúde dos motoristas de ônibus urbanos de Belo Horizonte.

\section{METODOLOGIA}

Trata-se de uma pesquisa transversal, realizada no período entre abril de 2017 a outubro de 2018, em estações de ônibus de Belo Horizonte, no estado de Minas Gerais, Brasil, onde existem sete estações de integração das quais quatro foram incluídas nesta pesquisa, pelo quantitativo elevado de motoristas, fato que viabilizou o maior contato e haver tempo de descanso para os profissionais. As demais estações são apenas áreas de circulação com embarque e desembarque de passageiros, mas sem tempo de descanso para os motoristas (BELO HORIZONTE, 2018).

A escolha dos motoristas de ônibus urbano de Belo Horizonte se deu pela técnica de amostragem por conveniência, sem determinação de uma linha de ônibus específica. Como critério de inclusão para o estudo, estabeleceu-se a participação de profissionais que tinham no mínimo um ano de experiência na profissão. A pesquisa foi apresentada verbalmente com assinatura do Termo de Consentimento Livre e Esclarecido (TCLE) pelos participantes.

As entrevistas foram realizadas por cinco acadêmicos de enfermagem devidamente treinados e, utilizou-se um questionário semiestruturado com questões objetivas e discursivasrelativas ao perfil socioeconômico e profissional, condições de saúde, hábitos alimentares, comportamentais dos entrevistados e aferição do Índice de Massa Corporal (IMC). A variável de desfecho, "autopercepção de saúde" foi separada dicotomicamente em "boa" e "ruim" para fins de análise. As respostas das questões, assim como as aferições, foram registradas ou assinaladas pelos entrevistadores.

Os dados coletados foram tabulados por meio do Microsoft Office Excel 2013, analisados descritiva e estatisticamente com o programa estatístico Data Analysis and Statistical Software (STATA) versão 14.0, utilizando-se os testes Qui-quadrado e Exato de Fisher. Para as variáveis como faixa etária, peso, tempo de profissão, salário, carga horária semanal, atividade física por semana, foram utilizadas a mediana e o desvio-padrão (DP) ou a mediana

Esta pesquisa foi aprovada por Comitê de Ética e Pesquisa, CAAE 73927517.8.0000.5137, seguindo as determinações da resolução 466/2012 do Conselho Nacional de Saúde.

\section{RESULTADOS}

Participaram da pesquisa 324 motoristas: $321(99,1 \%)$ do sexo masculino e $3(0,9 \%)$ do sexo feminino; idade média de 41 anos (26-66 anos); 157 (48,5\%) afirmaram serem pardos; 219 (67,6\%) relataram ser casados; 191 (59\%) declararam ter ensino Médio e a maioria $(n=111 / 34,3 \%)$ informou ter dois filhos. Demais dados sociodemográficos estão na Tabela 01. 
Tabela 01 - Perfil sociodemográficos dos motoristas de ônibus de Belo Horizonte - MG, n=324 , 2018.

\begin{tabular}{|c|c|c|c|}
\hline Variáveis & Categorias & $\mathbf{n}$ & $\%$ \\
\hline \multirow{2}{*}{ Idade (mediana) } & $\leq 41$ & 162 & 50,0 \\
\hline & $>41$ & 162 & 50,0 \\
\hline \multirow{2}{*}{$\begin{array}{l}\text { Peso referido } \\
\text { (mediana) }\end{array}$} & $\leq 80$ & & \\
\hline & $>80$ & & \\
\hline \multirow{2}{*}{ Sexo } & Masculino & 321 & 99,1 \\
\hline & Feminino & 3 & 0,9 \\
\hline \multirow{4}{*}{ Cor de pele } & Parda & 157 & 48,5 \\
\hline & Branca & 88 & 27,2 \\
\hline & Negra & 74 & 22,8 \\
\hline & Amarela & 5 & 1,5 \\
\hline \multirow{4}{*}{ Estado civil } & Casado & 219 & 67,6 \\
\hline & Solteiro & 52 & 16,1 \\
\hline & Divorciado/Viúvo & 41 & 13,0 \\
\hline & Outros & 12 & 3,7 \\
\hline \multirow{5}{*}{ Escolaridade } & Sabe Ler/Escrever & 1 & 0,3 \\
\hline & Ensino Fundamental Completo/Incompleto & 97 & 29,9 \\
\hline & Ensino Médio Completo/Incompleto & 191 & 59,0 \\
\hline & Nível Técnico & 10 & 3,1 \\
\hline & Superior Completo/Incompleto & 25 & 7,7 \\
\hline \multirow{3}{*}{ Mora com } & Família (pais, cônjuge, filhos, irmãos) & 286 & 88,3 \\
\hline & Sozinho & 26 & 8,0 \\
\hline & Amigos/República/outros & 12 & 3,7 \\
\hline \multirow{4}{*}{ Número de filhos } & Nenhum & 40 & 12,4 \\
\hline & Um & 98 & 30,3 \\
\hline & Dois & 111 & 34,3 \\
\hline & $\geq$ Três & 75 & 23,2 \\
\hline \multirow{3}{*}{ Situação de Moradia } & Própria & 257 & 79,3 \\
\hline & Alugada & 49 & 15,1 \\
\hline & Cedida/Favor & 18 & 5,6 \\
\hline
\end{tabular}

Fonte: Dados da pesquisa, 2018.

Em relação ao trabalho, o tempo médio de serviço foi de 11 anos (6-20 anos). Cerca de 317 (97,8\%) relataram trabalhar durante o fim de semana e 361 (97,5\%) afirmaram terem quatro folgas ou mais ao longo do mês. A carga horária semanal dos entrevistados foi, em média, de 47 horas (42-48 horas) e a média salarial foi de $\mathrm{R} \$ 2.180,00$ (R\$2.100,00-R\$2.400,00). Outros dados são apresentados na Tabela 02. 
Tabela 02 - Perfil dos motoristas de Belo Horizonte - MG, de acordo com as variáveis laborais. n= 324, 2018.

\begin{tabular}{lccc}
\hline \multicolumn{1}{c}{ Variáveis } & Categorias & N & $\%$ \\
\hline \multirow{2}{*}{ Tempo de serviço (mediana/anos) } & $\leq 11$ & 163 & 50,3 \\
& $>11$ & 161 & 49,7 \\
\multirow{2}{*}{ Motorista no fim de semana } & Sim & 317 & 97,8 \\
\hline \multirow{2}{*}{ Folgas no mês } & Não & 7 & 2,2 \\
\hline \multirow{2}{*}{ Carga horária semanal (mediana/horas) } & 1 a 3 & 8 & 2,5 \\
& $\geq 4$ & 361 & 97,5 \\
\hline \multirow{2}{*}{ Salário (mediana/reais) } & $>47$ & 162 & 50,0 \\
& $>47$ & 162 & 50,0 \\
\hline
\end{tabular}

Fonte: Dados da pesquisa, 2018.

A variável "autopercepção de saúde" foi dicotomizada em "autopercepção de saúde boa" ou "autopercepção de saúde ruim". A maioria dos motoristas ( $n=234 / 72,8 \%)$ definiu a própria saúde como "boa" e 88 (27,2\%), como "ruim".

Em relação às doenças crônicas, $260(80,3 \%)$ relataram não possuir e $64(19,8 \%)$ afirmaram a ocorrência de alguma doença crônica.

Na entrevista, 247 (76,2\%) afirmaram possuir queixas relacionadas ao trabalho; muitos trabalhadores afirmaram sentir algum desconforto musculoesquelético, sendo o maior índice para dores nas costas ( $n=173 / 53,4 \%$ ), e dor de cabeça e em membros inferiores na mesma proporção ( $n=165 / 50,9 \%)$. Ademais, foi questionado aos entrevistados sobre suas queixas e desconfortos relacionados ao ambiente e convívio de trabalho, apresentado na Tabela 03.

O hábito de urinar durante o trabalho variou entre 1 a 2 vezes para $70(21,6 \%)$ motoristas; a partir de 3 , para 240 (74,1\%); e 14 (4,3\%) afirmaram não urinar durante o trabalho.

A maioria dos motoristas ( $n=272 / 84 \%$ ) informou que não eram fumantes, contudo, 139 participantes (43\%) se identificaram como fumantes passivos. Na realização de atividades físicas, 164 (50,6\%) dos motoristas informaram não realizar nenhuma atividade física durante a semana. Com relação ao sono, $147(45,4 \%)$ não se sentem descansados após dormirem e 164 (50,6\%) percebem um descanso parcial. No IMC aferido dos participantes da pesquisa, obteve-se baixo peso $(n=1 / 0,3 \%)$, normal $(n=118 / 36,45)$, sobrepeso $(n=141 / 43,5 \%)$ e obeso $(n=64 / 19,8 \%)$.

No tocante à satisfação com o trabalho, a maioria apresenta satisfação em todos os itens investigados (gerência; com o trabalho em si; colegas; passageiros), variando entre 95,1\% (satisfação com colegas) a 69,4\% (satisfação com passageiros).

No que tange à procura pelos serviços de saúde, no último ano a partir da data da entrevista, $130(40,1 \%)$ motoristas relataram que procuraram o centro de saúde, pelo menos, uma ou duas vezes, nos últimos 12 meses; e 226 (82\%) afirmaram que suas demandas foram solucionadas. A procura pelo serviço de saúde público correspondeu a 14 (4,3\%) para as unidades básicas de saúde e 22 (6,8\%) para as unidades de pronto atendimento (UPA). 0 setor privado foi utilizado por 149 (46\%) motoristas no pronto-socorro hospitalar e 134 (41,4\%), em clínicas ou consultórios. Dos pesquisados, seis $(1,8 \%)$ buscaram apoio no consultório da empresa e os demais em farmácia. 
A Tabela 03 apresenta o resultado da análise bivariada entre as variáveis laborais e relativas à saúde com a variável-resposta dicotomizada, destacando-se aquelas que apresentaram associação $(p<0,05)$. Motoristas que não possuíam doenças crônicas $(n=203 / 78,1 \%)$, não utilizavam medicação contínua ( $n=195 / 77,4 \%$ ) e não eram fumantes ( $n=207 / 76,1 \%)$ apresentaram uma boa autopercepção de saúde. Motoristas que possuíam doenças crônicas ( $n=31 / 48,4 \%)$, utilizavam medicação contínua $(n=31 / 43,1 \%)$, apresentaram queixas relacionadas ao trabalho $(n=81 / 32,8 \%)$ e relataram piora dos hábitos alimentares após ingressarem na profissão( $n=53 / 33,5 \%)$, apresentaram uma percepção de saúde ruim. 
Tabela 03-Variáveis laborais e de saúde associadas à autopercepção de saúde dos motoristas $(n=324)$ de Belo Horizonte - MG, 2018.

\begin{tabular}{|c|c|c|c|}
\hline \multirow{3}{*}{ Variáveis } & \multicolumn{2}{|c|}{ Autopercepção de Saúde } & \multirow{3}{*}{$P$ Valor } \\
\hline & Boa & Ruim & \\
\hline & n (\%) & n (\%) & \\
\hline Possui doença crônica & & & 0,000 \\
\hline Sim & $33(51,6)$ & $31(48,4)$ & \\
\hline Não & $203(78,1)$ & $57(21,9)$ & \\
\hline Hipertensão arterial sistêmica & & & 0,000 \\
\hline Sim & $25(51,0)$ & $24(49,0)$ & \\
\hline Não & $211(76,7)$ & $64(23,3)$ & \\
\hline Asma & & & 0,000 \\
\hline Sim & $05(83,3)$ & $01(16,6)$ & \\
\hline Não & $231(72,6)$ & $87(27,4)$ & \\
\hline Diabetes Mellitus & & & 0,001 \\
\hline Sim & $08(40,0)$ & $12(60,0)$ & \\
\hline Não & $227(74,9)$ & $76(25,1)$ & \\
\hline Utiliza medicação contínua & & & 0,001 \\
\hline Sim & $41(56,9)$ & $31(43,1)$ & \\
\hline Não & $195(77,4)$ & $57(22,6)$ & \\
\hline Queixas relacionadas ao trabalho & & & 0,000 \\
\hline Sim & $166(67,2)$ & $81(32,8)$ & \\
\hline Não & $70(90,9)$ & $07(9,1)$ & \\
\hline Dor de cabeça & & & 0,000 \\
\hline Sim & $103(62,4)$ & $62(37,6)$ & \\
\hline Não & $133(83,7)$ & $26(16,4)$ & \\
\hline \multicolumn{4}{|l|}{ Dor nas costas } \\
\hline Sim & $114(65,9)$ & $59(34,1)$ & \\
\hline Não & $122(80,8)$ & $29(19,2)$ & \\
\hline Dores nos membros inferiores & & & 0,000 \\
\hline Sim & $102(61,8)$ & $63(38,2)$ & \\
\hline Não & $134(84,3)$ & $25(15,7)$ & \\
\hline Dores musculoesqueléticas & & & 0,000 \\
\hline Sim & $101(63,5)$ & $58(36,5)$ & \\
\hline Não & $135(81,8)$ & $30(18,2)$ & \\
\hline Ouve bem & & & 0,000 \\
\hline Sim & $200(77,5)$ & $58(22,5)$ & \\
\hline Não & $36(54,6)$ & $30(45,4)$ & \\
\hline Queixa de zumbidos & & & 0,000 \\
\hline Sim & $36(55,4)$ & $29(44,6)$ & \\
\hline
\end{tabular}




\begin{tabular}{lccc} 
Não & $200(77,2)$ & $59(22,8)$ & 0,000 \\
\hline Satisfação com empresa & & $55(21,3)$ & \\
\hline Sim & $203(78,7)$ & $32(49,2)$ & \\
Não & $33(50,8)$ & & 0,028 \\
\hline Satisfação com passageiros & & $53(23,6)$ & \\
\hline Sim & $172(76,4)$ & $35(35,4)$ & 0,003 \\
Não & $64(64,6)$ & & \\
\hline Fumante & & $23(44,2)$ & 0,001 \\
\hline Sim & $29(55,8)$ & $65(23,9)$ & \\
Não & $207(76,1)$ & & \\
\hline Hábitos alimentares após ingressar & & $30(25,6)$ & \\
na profissão & & $53(33,5)$ & \\
\hline Melhorou & $87(74,4)$ & $05(10,2)$ & \\
Piorou & $105(66,5)$ & $22(39,3)$ & \\
\hline Não modificou & $44(89,8)$ & & \\
\hline Almoço de qualidade & & & \\
\hline Sim & $201(75,4)$ & $(60,2)$ & \\
\hline Não & & & \\
\hline
\end{tabular}

Fonte: Dados da pesquisa, 2018.

$\mathrm{Na}$ análise ajustada (tabela/número), não possuir hipertensão arterial sistêmica, dor de cabeça, dores em membros inferiores, estar satisfeito com a empresa e não ser fumante associou-se à boa autopercepção de saúde, em contrapartida com o prejuízo da audição (Tabela 4). 
Tabela 4 -Modelo de regressão multivariada de Poisson, ajustado para os fatores associados à boa autopercepção de saúde entre os motoristas $(N$ = 324) de Belo Horizonte - MG, 2018.

\begin{tabular}{|c|c|c|}
\hline Variáveis & RP (IC 95\%) & Valor de $p$ \\
\hline Hipertensão arterial sistêmica & & 0,004 \\
\hline Sim & - & \\
\hline Não & $1,5(1,1-1,9)$ & \\
\hline Cefaleia & & 0,013 \\
\hline Sim & - & \\
\hline Não & $1,2(1,0-1,3)$ & \\
\hline Dores em membros inferiores & & 0,002 \\
\hline Sim & - & \\
\hline Não & $1,2(1,1-1,4)$ & \\
\hline Ouve bem & & 0,008 \\
\hline Sim & - & \\
\hline Não & $0,7(0,6-0,9)$ & \\
\hline Satisfação com dono da empresa & & 0,003 \\
\hline Sim & $1,4(1,1-1,8)$ & \\
\hline Não & - & \\
\hline Fumante & & 0,033 \\
\hline Sim & - & \\
\hline Não & $1,3(1,0-1,6)$ & \\
\hline
\end{tabular}

Fonte: Dados da pesquisa, 2018.

\section{DISCUSSÃO}

Em relação ao perfil sociodemográfico, trata-se de uma classe trabalhadora na qual predomina o sexo masculino, estado civil casado e com ensino médio, corroborando com dados existentes na literatura (MOURA; SILVA, 2012; GONÇALVES et al., 2014; ASSUNÇÃO; PIMENTA, 2015). Em contrapartida, estudos com um quantitativo menor indicaram uma prevalência de profissionais com ensino fundamental de $85,7 \%$ e $61,8 \%$, sobrepondo o encontrado nesta pesquisa (BATTISTON; CRUZ; HOFFMANN, 2006; DE VITTA et al., 2013). Infere-se que a prevalência de trabalhadores com ensino médio é devido aos pré-requisitos das atuais empresas de transporte coletivo para pleitear a vaga.

Em relação ao tempo médio de profissão cerca da metade dos participantes revelou mais de onze anos na profissão, o que corrobora com pesquisa em dois municípios do Estado do Rio Grande do Sul, com $41 \%$ a $60 \%$ dos motoristas de ônibus com, pelo menos, dez anos na função (BENVEGNú et al. 2008; MOURA; SILVA, 2012).

$\mathrm{Na}$ indagação acerca de sintomas de mal-estar físico, de lombalgias, cefaleia e dor nos membros inferiores, mais da metade dos condutores relataram a presença delas. As dores nos membros inferiores tiveram relação estatisticamente significativa (análise multivariada) com a autopercepção de saúde. Outra pesquisa encontrou $65,7 \%$ dos motoristas participantes com alguma queixa musculoesquelética, sendo mais frequentes na região lombar, torácica e ombros (BATTISTON; CRUZ; HOFFMANN, 2006; DE VITTA et al., 2013; ASSUNÇÃO; PIMENTA, 2015). São resultados justificados pela carga de trabalho por longo período e por atividades repetitivas envolvendo troca de marchas e disposição no volante dos veículos (DE VITTA et al., 2013). 
Uma variável relevante, quando se avalia a saúde ocupacional de motoristas de ônibus, é o ruído advindo dos motores alocados na parte dianteira dos veículos, do ambiente externo e dos passageiros. Apesar da maioria dos trabalhadores terem considerado o ruído como elevado ou insuportável, também referiu boa audição e ausência de zumbidos diferentemente de outro estudo que detectou alterações auditivas (BATTISTON; CRUZ; HOFFMANN, 2006; ASSUNÇÃO; PIMENTA, 2015).

No que tange a doenças crônicas, a maioria dos entrevistados negou ser acometido e, entre aqueles que afirmaram possuir, a hipertensão arterial sistêmica (HAS) teve destaque, sendo também confirmada pela literatura (ALQUIMIM et al., 2012). Sabe-se que, a longo prazo, a hipertensão arterial causa diversos prejuízos ao organismo do indivíduo, acarretando uma piora do seu estado de saúde, tornando-se fator de agravo para uma visão de saúde ruim, como observado na presente pesquisa (ASSUNÇÃO; PIMENTA, 2015).

Nesta pesquisa, o IMC avaliado, com altura e peso autodeclarados, demonstrou que $63,3 \%$ dos motoristas apresentavam alteração em sobrepeso ou obesidade, sendo confirmado entre motoristas em outras pesquisas (CHAVES et al., 2008; HIRATA et al., 2011; ALQUIMIM et al., 2012; MOURA; SILVA, 2012; SOUZA; ASSUNÇÃO; PIMENTA, 2019). Esse dado é acompanhado pela ausência de atividade física e pela alimentação inadequada e, 50,6\% dos entrevistados não realizavam atividade física, o que sobressaiu frente a outro estudo (43,6\%), com associação estatisticamente significativa para o desenvolvimento de sintomas musculoesqueléticos ( $p<0,001$ ). (DE VITTA et al., 2013; ASSUNÇÃO; PIMENTA, 2015).

Os hábitos alimentares de 48,8\% pioraram após o ingresso na profissão, com tendência de maior consumo de lanches e salgados rápidos a partir do ingresso na categoria, pois é de consumo rápido e fácil acesso (ALQUIMIM et al, 2012). Ressalta-se que a alimentação inadequada e inatividade física podem levar ao aumento de peso do trabalhador, sendo fatores de risco para doenças cardiovasculares (HIRATA et al., 2011; MORAES; FAYH, 2011).

Os tabagistas identificados na pesquisa corresponderam a somente $16,1 \%$ dos motoristas, resultado semelhante com o encontrado em outros estudos (MORAES; FAYH, 2011; MOURA; SILVA, 2012; ASSUNÇÃO; PIMENTA, 2015). A condição de não fumante, nesta pesquisa, foi um fator associado a boa autopercepção de saúde ( $R C=1,3 ; 95 \% \mathrm{IC}: 1,0-1,6 ; p=0,033)$. 0 inquérito de Vigilância de Fatores de Risco para Doenças Crônicas (Vigitel), conduzido pelo Ministério da Saúde, apontou, em 2017, a frequência de fumantes de 4,1\% em Salvador e 15,6\% em Curitiba (BRASIL, 2019). 0 menor índice de fumantes pode ser indicativo de programas e leis desenvolvidas no país para reduzir a utilização do tabaco (ALQUIMIM et al., 2012). Em pesquisa realizada em Buenos Aires, indicou que $49 \%$ dos entrevistados eram tabagistas (PERES et al., 2010).

Outro estudo no mesmo município desta pesquisa, contudo com motoristas e acompanhantes de bordo dos transportes coletivos, apontou que os critérios ser do sexo feminino, ter idade entre 30-49 anos, ter filhos, ser ex-fumante, não praticar atividade física, ter diagnóstico médico de insônia tiveram associação e se relacionaram à obesidade em nível bivariado (SOUZA; ASSUNÇÃO; PIMENTA, 2019).

A maioria dos motoristas relatou uma boa autopercepção de saúde $(72,8 \%)$, sendo referida pela maioria dos condutores que não possuíam doenças crônicas, não utilizava medicações contínuas, sem queixas musculoesqueléticas e não fumantes. Em pesquisa com a autoavaliação de saúde em adultos da região sul do país, a autoavaliação positiva em maiores proporções foi apontada pelos participantes com maiores rendas, mais escolarizados, mais jovens e de cor branca ou amarela (PERES et al, 2010); em outra, os participantes informaram que $58,9 \%$ dos motoristas possuíam uma boa percepção de saúde e 26,2\%, percepção "excelente/muito boa" (MOURA; SILVA, 2012); no estudo com motoristas e cobradores, a autoavaliação "ruim/muito ruim" de saúde foi estatisticamente significativa com a 
prevalência de distúrbios psiquiátricos entre trabalhadores do transporte coletivo $(R C=1,53 ; 95 \%$ IC: 1,28-1,83; $p<0,01$ ) (ASSUNÇÃO; SILVA, 2013).

\section{CONCLUSÃo}

Conclui-se que os motoristas apresentam uma boa autopercepção de saúde, apesar de reconhecerem fatores laborais de hábitos e ambientais desfavoráveis. Ademais, eles possuem limitações na percepção de saúde e no conceito de saúde como sendo a ausência de doença. Entende-se, portanto, que para tal categoria profissional, ações de promoção de saúde são relevantes no sentido de estimular o autocuidado, a prevenção de agravos frequentemente identificados nesses trabalhadores e evidenciados pela literatura. Discussões também no nível da melhoria da gestão e organização do trabalho serão propulsoras para o bem estar dessas pessoas.

Como limitações da pesquisa, aponta-se que alguns dados foram de auto relato não sendo realizadas medições mais precisas, assim como da amostra por conveniência.

\section{AGRADECIMENTOS}

Ao Sindicato dos Trabalhadores em Transportes Rodoviários de Belo Horizonte e Região - STTRBH pela parceria e apoio à pesquisa.

\section{REFERÊNCIAS}

ALQUIMIM, A.F.; BARRAL, A.B.C.R.; GOMES, K.C.; REZENDE, M.C. Avaliação dos fatores de risco laborais e físicos para doenças cardiovasculares em motoristas de transporte urbano de ônibus em Montes Claros (MG). Ciência e Saúde Coletiva, v. 17, n. 8, 2012. Disponível em: http://scielo.br/scielo.php?script=sci_ arttext\&pid=S1413-81232012000800025. Acesso em: 15 nov. 2019.

ALVES, C.R.S.; PAULA, P.P. Violência no trabalho: possíveis relações entre assaltos e TEPT em rodoviários de uma empresa de transporte coletivo. São Paulo. Cadernos de Psicologia Social do Trabalho, v. 12, n. 1, p. 35-46, 2009. Disponível em: http://pepsic.bvsalud.org/scielo.php?script=sci_ arttext\&pid=S151637172009000100004\&lng=pt\&nrm=iso. Acesso em: 22 out. 2019

ASSUNÇÃO, A.A.; MEDEIROS, A.M. Violência a motoristas e cobradores de ônibus metropolitanos, São Paulo. Revista de Saúde Pública, v. 49, n. 11, p. 1-10, 2015. Disponível em: http://www.scielo.br/scielo. php?pid=S0034-89102015000100207\&script=sci_arttext\&tlng=pt. Acesso em: 19 out. 2019.

ASSUNÇÃO, A.A.; PIMENTA, A.M.A. Exposição à vibração e a hipertensão arterial em trabalhadores do transporte coletivo metropolitano. Revista Brasileira de Saúde Ocupacional, v. 40, n. 132, p. 196-205, 2015. Disponível em: http://www.scielo.br/pdf/rbso/v40n132/0303-7657-rbso-40-132-196.pdf. Acesso em: 15 dez. 2019.

ASSUNÇÃO, A.A.; SILVA, L.S. Condições de trabalho nos ônibus e os transtornos mentais comuns em motoristas e cobradores: região metropolitana de Belo Horizonte, Minas Gerais, Brasil. Cadernos de Saúde Pública, v. 29, n. 12, p. 2473-2486, 2013. Disponível em: http://www.scielo.br/scielo.php?script=sci_ abstract\&pid=S0102-311X2013001200012\&lng=en\&nrm=iso\&tlng=pt. Acesso em: 29 set. 2019. 
AZEVEDO, G.P.C.; FRICHE, A.A.L.; LEMOS, S.M.A. Autopercepção de saúde e qualidade de vida de usuários de um ambulatório de fonoaudiologia. Revista da Sociedade Brasileira de Fonoaudiologia, v. 17, n. 2, p. 119-127, 2012. Disponível em: http://www.scielo.br/scielo.php?script=sci_arttext\&pid=S1516-80342012000200004. Acesso em: 20 ago. 2019.

BATTISTON, M.; CRUZ, R.M.; HOFFMANN, M.H. Condições de trabalho e saúde de motoristas de transporte coletivo urbano. Estudos de Psicologia, v. 11, n. 3, p. 333-343, 2006. Disponível em: http://www.scielo. br/scielo.php?script=sci_arttext\&pid=S1413-294X2006000300011. Acesso em: 09 nov. 2019.

BELO HORIZONTE. BHTRANS. Estações: estações de integração. 2018. Disponível em: https://prefeitura. pbh.gov.br/bhtrans/informacoes/transportes/onibus/estacoes. Acesso em: 10 out. 2019.

BENVEGNÚ, L.A.; FASSA, A.G.; FACCHINI, L.A.; BREITENBACH, F. Prevalência de hipertensão arterial entre motoristas de ônibus em Santa IMaria, Rio Grande do Sul. Revista Brasileira de Saúde Ocupacional, v. 33, n. 118, 2008. Disponível em: http://www.scielo.br/scielo.php?script=sci_ arttext\&pid=S0303-76572008000200004. Acesso em: 2 ago. 2019.

BEZERRA, S.M.; SILVA, E.F.; MARIBONDO, J.F.; PINTO, F.M. A influência das condições e organização do trabalho sobre a saúde de motoristas. Revista eletrônica Ciências, v. 11, n. 16, p. 1-11, 2011. Disponível em: http://revistatema.facisa.edu.br/index.php/revistatema/article/view/75/83. Acesso em: 15 ago. 2019.

BRASIL. Decreto-Lei n. 8.069 de 13 de julho de 1990. Dispões sobre o Estatuto da Criança e do Adolescente e dá outras providências. Diário Oficial da União, Brasília, 13 jul. 1990. Disponível em: http://www. planalto.gov.br/ccivil_03/leis/L8069.htm. Acesso em: 02 jun. 2019.

BRASIL. Ministério da Saúde. Secretaria de Vigilância em Saúde. Departamento de Vigilância de Doenças e Agravos não Transmissíveis e Promoção da Saúde. Vigitel Brasil 2017: vigilância de fatores de risco e proteção para doenças crônicas por inquérito telefônico: estimativas sobre frequência e distribuição sociodemográfica de fatores de risco e proteção para doenças crônicas nas capitais dos 26 estados brasileiros e no Distrito Federal em 2017 - Brasília: Ministério da Saúde, 2019. Disponível em: http:// bvsms.saude.gov.br/bvs/publicacoes/vigitel_brasil_2017_vigilancia_fatores_risco_1 ed_rev.pdf.. Acesso em: 09 fev. 2019.

CHAVES, D.B.R.; COSTA, A.G.S.; OLIVEIRA, A.R.S.; OLIVEIRA, T.C.; ARAÚJO, T.L.; LOPES, M.V.O. Fatores de risco para hipertensão arterial: investigação em motoristas e cobradores de ônibus. Revista de Enfermagem da UERJ, v.16, n.3, 2008. Disponível em: http://www.facenf.uerj.br/v16n3/v16n3a12.pdf. Acesso em: 15 nov. 2019.

DE VITTA, A.; DE CONTI, M.H.S.; TRIZE, D.M.; QUINTINO, N.M.; PALMA, R.; SIMEÃO, S.F.A.P. Sintomas musculoesqueléticos em motoristas de ônibus: prevalência e fatores associados. Fisioterapia em Movimento, v. 26, n. 4, p. 863-871, 2013. Disponível em: http://www.scielo.br/scielo.php?script=sci_ arttext\&pid=S0103-51502013000400015. Acesso em: 15 set. 2019.

DIEZ, J.; VIGO, E.D.; CARDINALI, P.D; PÉREZ-CHADA, D. Sleep habits, daytime sleepiness and working conditions in short-distance bus drivers. International Journal Workplace Health Management, v. 7, n. 4, p. 202-212, 2014. Disponível em: https://www.researchgate.net/publication/275043602_Sleep_habits_ daytime_sleepiness_and_working_conditions_in_short-distance_bus_drivers. Acesso em: 5 jun. 2019.

GONÇALVES, E.S.; TORRES, R.M.; PEIXINHO, T.C.; BORGES, C.C.L. Fatores de risco para doença arterial coronária em motorista de ônibus. Revista Brasileira de Enfermagem. v. 28, n. 3, p. 252-259, 2014 
Disponível em: https://portalseer.ufba.br/index.php/enfermagem/article/view/11399/8987. Acesso em: 20 set. 2019.

HIRATA, R.P.; CERRA, J.C.; MACEDO, C.R.; FAVARETO, J.; FILHO, F.S.S.L.; INSALACO, G.; OLIVEIRA, L.V.F.O. Prevalência de obesidade e hipertensão arterial em uma população de motoristas profissionais rodoviários interestaduais de ônibus. ConScientia Saúde, v. 10, n. 3, p. 494-499, 2011. Disponível em: https://periodicos.uninove.br/saude/article/view/3046. Acesso em: 22 ago. 2019.

MORAES, G.N.; FAYH, A.P.T. Avaliação nutricional e fatores de risco cardiovascular em motoristas de transporte coletivo urbano. Caderno de Saúde Coletiva, v. 19, n. 3, p.334-340, 2011. Disponível em: http://www.cadernos.iesc.ufrj.br/cadernos/images/csc/2011_3/artigos/csc_v19n3_334-340.pdf. Acesso em: 15 set. 2019.

MOURA, N.A.B.; SILVA, M.C. Diagnóstico das condições de trabalhos, saúde e indicadores do estilo de vida de trabalhadores do transporte coletivo da cidade de Pelotas - RS. Revista Brasileira de Atividade Física \& Saúde, v. 17, n. 5, p. 347-358, 2012. Disponível em: http://rbafs.org.br/RBAFS/article/view/2174/ pdf6. Acesso em: 15 jun. 2019.

OROZCO-SOLIS, M.G.; COLUNGA-RODRÍGUEZ, C.; PRECIADO-SERRANO, M.L.; GONZÁLEZ, M.A.; VÁZQUEZCOLUNGA, J.C.; COLUNGA-RODRÍGUEZ, B.A. Representation of self-care of health at work among urban bus drivers in Guadalajara, Mexico. Caderno de Saúde Pública, v. 33, n. 3, p.1-13, 2017. Disponível em: http://www.scielo.br/pdf/csp/v33n3/1678-4464-csp-33-03-e00139815.pdf. Acesso em: 5 jun. 2019.

PAVÃO, A.L.B.; WERNECK, G.L.; CAMPOS, M.R. Autoavaliação do estado de saúde e a associação com fatores sociodemográficos, hábitos de vida e morbidade na população: um inquérito nacional. Cadernos de Saúde Pública; v. 29, n. 4, 2013, p. 723-734. Disponível em: http://www.scielo.br/scielo. php?pid=S0102-311X2013000400010\&script=sci_abstract\&tlng=pt. Acesso em: 03 set. 2019.

PERES, M.; MASIERO, V.; LONGO, G.Z.; ROCHA, G.C.; MATOS, I.B.; NAJNIE, K.; OLIVEIRA, M.C.; ARRUDA, M.P.; PERES, K.G. Autoavaliação da saúde em adultos no Sul do Brasil. Revista de Saúde Pública, v. 44, n. 5, p. 901-911, 2010. Disponível em: http://www.scielo.br/pdf/rsp/v44n5/1283.pdf. Acesso em: 10 out. 2019.

PINTO, F.M.; NEVES, M.Y. A gestão da atividade do motorista de ônibus: um olhar ergológico. Estudos e Pesquisas em Psicologia, v. 9, n. 2, p. 493-511, 2009. Disponível em: http://www.revispsi.uerj.br/v9n2/ artigos/pdf/v9n2a15.pdf. Acesso em: 10 jun. 2019.

SOUZA, L.P.S.; ASSUNÇÃO, A.A.; PIMENTA, A.M. Fatores associados à obesidade em rodoviários da Região Metropolitana de Belo Horizonte, Minas Gerais, Brasil. Revista Brasileira de Epidemiologia, v. 22, p. 1-15, 2019. Disponível em: http://www.scielo.br/scielo.php?script=sci_arttext\&pid=S1415790X2019000100424\&Ing=pt. Acesso em: 08 jan. 2020.

VASCONCELLOS, E.A.; CARVALHO, C.H.R.; PEREIRA, R.H.M. Transporte e mobilidade urbana. Brasília, Distrito Federal: CEPAL. Escritório no Brasil/IPEA, 2011. Texto para Discussão, CEPAL-IPEA, 34. Disponível em: http://repositorio.ipea.gov.br/bitstream/1 1058/1373/1/TD_1552.pdf.. Acesso em: 08 jan. 2019. 